\title{
EL ALUMNADO CON DIVERSIDAD FUNCIONAL EN LA UNIVERSIDAD DE SEVILLA: RESULTADOS DE UN ESTUDIO BIOGRÁFICO-NARRATIVO
}

\section{Almudena Cotán Fernández}

\section{RESUMEN}

El artículo que presentamos se encuentra enmarcado dentro de un proyecto de investigación', actualmente en desarrollo y desde un enfoque biográfico-narrativo, cuyo objetivo principal se centra en identificar $y$ estudiar las barreras $y$ ayudas que los estudiantes con diversidad funcional encuentran en la Universidad de Sevilla. Aunque, en el proceso de investigación mencionamos evidencias de lo que ocurre en los cinco campos de conocimiento, en este artículo sólo nos centraremos en las voces de un grupo de estudiantes de las Facultades de Ciencias de la Educación, Psicología y Filosofía. Los resultados que aquí se presentan pertenecen a la primera fase del proyecto de investigación; en concreto, nos centraremos en las barreras $y$ a las ayudas que los estudiantes con diversidad funcional identifican en el profesorado y en sus metodologías durante su trayectoria universitaria.

Palabras clave: alumnado con diversidad funcional, Enseñanza Universitaria, Educación Inclusiva, Exclusión Educativa, Profesorado.

\section{TITLE: STUDENTS WITH DISABILITIES AT THE UNIVERSITY OF SEVILLE: RESULTS OF A BIOGRAPHICAL-NARRATIVE STUDY}

\section{ABSTRACT}

The article we present is framed within a research project' ${ }^{\prime}$ currently underway and is described using a biographical-narrative approach, the main objective of which is focused on identifying and studying the barriers and support students with disabilities find in the University of Seville. Although in the research process, we mention evidence of what is happening in the five fields of knowledge, in this article we focus only on the information provided by a group of students from the faculties of Education, Psychology and Philosophy. The results presented here belong to the first phase of the research project; in particular, we will focus on the barriers and support students with disabilities identified among faculty and the methodologies used during their university courses.

Keywords: Students with disabilities, University Education, Inclusive Education, Educational Exclusion, Faculty.

Correspondencia con la autora: Almudena Cotán Fernández. < acotan@ceuandalucia.es > Centro de Estudios Universitarios Cardenal Spínola CEU. Original recibido: 02-09-13. Original aceptado: 26-02-14 


\section{Introducción}

Durante los últimos años, el acceso de estudiantes con diversidad funcional en la Universidad de Sevilla ha notado un importante aumento, llegando a la cifra de 500 alumnos aproximadamente durante el curso $09 / 10^{2}$; una realidad similar que está ocurriendo en otros contextos universitarios nacionales e internacionales (Barnes, 2008; Novo, Cantero y Calvo, 20 I I).

En la actualidad, y debido al cambio de Plan de estudios con el proceso de Bolonia, las universidades están participando en un proceso de reflexión e innovación con un objetivo común: garantizar la calidad y equidad de sus procesos formativos y servicios educativos mediante una cultura de evaluación y estrategias de auto-regulación. Ello, unido a los más de 8000 alumnos y alumnas con diversidad funcional que estudian en las universidades españolas, (un $0,5 \%$ del total del alumnado matriculado), nos ha conducido a considerar imprescindible una reflexión sobre las barreras y ayudas que los estudiantes con diversidad funcional, se encuentran y enfrentan a lo largo de su trayectoria académica en la Universidad de Sevilla ${ }^{3}$ en el ámbito de aula, más concretamente en relación al profesorado y sus proyectos docentes.

Existen diversos trabajos (Castellana y Sala, 2005; Sánchez Palomino, 2009, 20II; Sánchez y Carrión, 2010) que señalan, las barreras de tipo actitudinal e institucional con las que se encuentra el alumnado con diversidad funcional en la Universidad, reflejando así el desconocimiento actual hacia la propia discapacidad, la falta de inclusión que sufren, y a veces, las respuestas negativas que reciben. También, en estos trabajos se reflejan las barreras relacionadas con el curriculum, como metodologías poco adecuadas, materiales inaccesibles o programaciones que homogeneizan. Asimismo, en estos estudios aparece la necesidad de que el profesorado se forme sobre este tema y, por lo tanto, se recomienda procesos de asesoramiento y formación sobre discapacidad y atención a la diversidad.

En cuanto a la perspectiva internacional (Borland y James, 1999; Gitlow, 1999; Healey, Fuller, Bradley y Hall, 2006; Moswela y Mukhopadhyay, 20I I; Parker, 1999; Poussu-Olli, 1999 o Vickerman y Blundell, 20I0; etc.), reafirman los procesos de exclusión y segregación experimentados y vividos por estos estudiantes debido a la falta de formación que tienen los docentes para atender a la inclusión.

Frente a los resultados de estas investigaciones, compartimos con autores como Moriña (2010), Ainscow (1999; 2004; 2008), Arnaiz (2003), Barton (1998; 2008) y Echeíta (2006), que uno de los primeros pasos que se ha de dar en el sector educativo, sería 
creer y asimilar que otra educación es posible, donde se dé respuesta a las necesidades del alumnado, el profesorado esté formado para atender a la diversidad de las aulas $y$, para ello, necesitaríamos de un cambio profundo (social, cultural, legislativo, educativo...) y continúo, donde siempre se esté en un constante movimiento hacia la inclusión y donde la comunidad escolar trabaje de manera colaborativa y cooperativa. No obstante, tal y como afirman Moriña y Parrillas (2007), la formación del profesorado nunca ha de ser un modelo único, fijo y estable, sino que éste deberá de ser adaptada a las necesidades individuales del sujeto y de la sociedad en la que se encuentra inmersa bajo la orientación práctica y el modelo de desarrollo y mejora de la enseñanza (Marcelo, 1995).

La nueva forma de vida ha conllevado a diferentes y diversas formas de aprendizaje por parte de los sujetos; procesos a los que los profesionales de la enseñanza han de adaptarse y no quedarse obsoletos (Marcelo, 200I). El proceso educativo ha de entenderse como un proceso formativo que dirige la formación social de las nuevas generaciones, las instruye, las desarrolla y las educa. Marcelo (200I), propone una formación a lo largo de la vida, donde el ser humano esté en constante contacto con el aprendizaje y no se quede estancado en una mera "formación inicial con fecha de caducidad" (Marcelo, 200 I, p.2).

Según la UNESCO (2008) la nueva tendencia en la formación debería basarse y centrarse en objetivos que orienten a los principios de formación permanente, otorgando importancia la formación a lo largo de la vida, y que ésta, a su vez, se encuentre basada en un modelo de competencias y en la economía, refiriéndose éste última a combinar la duración de los procesos de aprendizaje con los programas formativos.

Respecto a las herramientas e instrumentos de enseñanza, Lavié y Marcelo (2000), indican que se ha de aprovechar el espacio que nos facilitan las Nuevas Tecnologías, ya que nos permiten hacer llegar una mayor cantidad de información en un corto espacio temporal, a un mayor números de personas, pudiendo trabajar juntas, desarrollar y mejorar su formación; y es en este nuevo espacio de teleformación, donde marcamos énfasis en la utilización de las Nuevas Tecnologías en los procesos de enseñanza-aprendizaje, en el aprendizaje autónomo por parte del estudiante y en el aprendizaje colaborativo y en grupo; todo ello, basándonos en un enfoque inclusivo, interdisciplinario e interdependiente, donde los currículos se encuentren adaptados a las necesidades de los estudiantes con diversidad funcional, mejorando su calidad de vida desde una postura de supresión de barreras físicas y mentales y, consiguiendo, por lo tanto, una verdadera y plena inclusión social dentro de la misma (Arana, Rodríguez y Meilán, 2008; Bausela, 2004; Novo, Cantero y Calvo, 20I I). 


\section{Diseño Metodológico de la Investigación}

El trabajo de investigación que presentamos a continuación, se encuentra vinculado al proyecto I+D+I "Análisis de las Barreras y Ayudas que los estudiantes con discapacidad identifican en la Universidad", con una duración de tres años (201 I2013). Dicho proyecto de investigación se está llevando a cabo por parte de un equipo multidisciplinar de la Universidad de Sevilla (Ciencias de la Educación, Ciencias Económicas, Ciencias de la Salud y Ciencias Experimentales).

El objetivo principal de la investigación pretende estudiar, desde la perspectiva de los estudiantes universitarios con diversidad funcional, las barreras y ayudas que identifican en su acceso, trayectoria y resultados en la universidad. Una de las metas planteadas en primer lugar, es analizar cómo la universidad como institución puede facilitar u obstaculizar los procesos formativos de estos estudiantes. En segundo lugar, estaríamos interesados en investigar cómo los procesos de enseñanza y aprendizaje, actitudes, relaciones, etc. que tienen lugar en las aulas universitarias pueden influir en sus procesos de aprendizaje y participación.

En este sentido, se propone identificar y explicar, dando voz a este grupo de estudiantes, mediante técnicas de investigación basadas en la metodología biográfico-narrativa, cuáles son los factores que perciben como obstáculos o ayudas en la universidad y en las aulas universitarias y por qué los identifican como tales. A partir de los datos recogidos, como fase final de la investigación, se diseñara una guía de formación online para que el profesorado universitario adquiera una serie de competencias necesarias para atender a la diversidad en las aulas.

Para responder a los objetivos propuestos desde una metodología biográficanarrativa, se han planteado tres fases de investigación. En la primera fase de recogida de datos, se ha realizado un análisis extensivo de las barreras y ayudas que los estudiantes con diversidad funcional vivencian en la universidad en general y en las aulas universitarias en particular. En esta fase, iniciamos la recogida de datos de los distintos grupos de discusión por los cinco campos de conocimiento ${ }^{4}$, como ya hemos mencionado anteriormente, grupos de discusión (uno o dos por cada área de conocimiento) y entrevistas individuales (orales y escritas). En esta fase han colaborado un total de 44 estudiantes.

En la segunda fase de investigación, se han realizado micro-historias de vida con un total de 9 estudiantes derivados de la fase anterior. La selección de estos estudiantes se ha realizado teniendo en cuenta varios criterios: idoneidad de la persona, historia narrada, motivación y dedicación a la investigación, entre otros. En esta fase las micro- 
historia de vida se caracterizan por ser temáticas (Sandín, 2003), ya que nos centramos en un período y asunto particular de la vida del estudiante que participa en esta investigación (su trayectoria universitaria). Las técnicas de recogida de datos empleadas en esta fase han sido tres: líneas de vida, entrevistas focalizadas y autoinformes.

En la tercera fase de la investigación, actualmente en desarrollo, hemos seguido desarrollando el proyecto con los estudiantes de la fase anterior realizando historias de vida en profundidad a través de la metodología biográfico-narrativa utilizando la polifonía de voces (Frank, 20I I). En esta ocasión, las técnicas de recogida de datos empleadas son los relatos cruzados (Pujadas, 2002), diarios, entrevistas biográficas y focalizadas, entrevistas a otros informantes (otras personas seleccionadas por el propio narrador o narradora y que contribuirán a construir su historia de vida) y fotografías.

Para responder al último objetivo de investigación ${ }^{5}$, se pretende realizar, cómo última fase de investigación, una propuesta de formación online para el profesorado de la universidad en relación a la diversidad.

El análisis de los datos se ha realizado a través de un sistema de codificación propuesto por Miles y Huberman (1994) y que se ha modificado tras la recogida de datos en la investigación "ya que el grupo final de categorías no está totalmente predeterminado sino que está compuesto por datos de acuerdo con los significados de categorías" (McMillan y Schumacher, 2005, p. 486).

A través de la codificación, hemos dividido los datos en 6 categorías generales: I. Información General; 2. Barreras Institucionales; 3. Ayudas Institucionales: 4. Barreras de Aula; 5. Ayudas de Aula; y 6. Prospectivas. Éstos a su vez, han sido divididos en subcategorías. No obstante, en este artículo únicamente nos centraremos en la primera, cuarta y quinta categoría.

A través de todas las transcripciones de los documentos generadas con las técnicas señaladas, se llevó a cabo un análisis comparativo de datos siguiendo el sistema de categorías creado y apoyándonos en el programa informático de análisis de datos Maxqda 10.

\section{Muestra}

La muestra total del trabajo $^{6}$ (primera fase) se encuentra compuesta por 44 estudiantes con diversidad funcional. En este artículo se analizarán I I experiencias y testimonios de estudiantes con diversidad funcional (tres chicos y ocho chicas) ${ }^{7}$ 
pertenecientes a las Facultades de Ciencias de la Educación, Psicología y Filosofía ${ }^{8}$, con edades comprendidas entre 19 y 39 años, situándose la media en 26 años, y con discapacidades física, psíquica y sensorial. De los II participantes, 6 personas se encuentran estudiando licenciaturas, 2 diplomaturas y otras dos se encuentran realizando estudios de postgrado. Tan sólo una de las participantes de la investigación había finalizado tanto sus estudios universitarios como de postgrado.

En relación a la población total de estudiantes que se presenta en este artículo, podemos decir que el $36,3 \%$ de estudiantes (cuatro) se encuentran en primero de carrera, el $18,2 \%$ (dos estudiantes) se encuentran en segundo, un $18,2 \%$ en tercero de carrera, un $9,1 \%$ (un estudiante) en cuatro, un $9,1 \%$ en quinto y un $9,1 \%$ que ha finalizado sus estudios. En relación a la media de años que han permanecido en la Universidad, la podemos situar en torno a los 5 años, convirtiéndose especialmente significativo, que un $36,3 \%$ de los estudiantes sitúen su media de estancia en la Universidad entre 8 y 10 años.

Por último, indicar que el tipo de discapacidad que los estudiantes reflejan y de acuerdo con la clasificación establecida en la Universidad de Sevilla, es la siguiente: $36,4 \%$ con discapacidad física, un $45,4 \%$ sensorial, un $9,1 \%$ psíquica y un $9,1 \%$ que posee tanto discapacidad sensorial como psíquica.

En este trabajo de investigación se garantizó en todo momento la negociación del consentimiento informado. Se dedicó un espacio para asegurarnos de que todos los participantes que aceptaron colaborar en la investigación comprendían el proceso en el que se iban a comprometer, incluyendo por qué su participación era necesaria, cómo se usaría la información y a quién llegaría. En la toma de contacto con los participantes, debemos de indicar que fue muy importante la labor del Servicio de Atención a la Comunidad Universitaria (SACU), a través de su unidad de Atención a las Personas con Discapacidad de la Universidad de Sevilla, puesto que a través de éste nos pusimos en contacto con los estudiantes y siempre rigiéndonos por la actual Política de Protección de datos ${ }^{7}$.

\section{Resultados}

En este apartado, como ya hemos explicado anteriormente, presentamos los resultados referidos exclusivamente a las barreras y ayudas que los estudiantes con diversidad funcional identifican en la Universidad de Sevilla, específicamente aquellos que hacen referencia a la actitud del profesorado y a sus experiencias con 
los proyectos docentes. En estos temas, abordaremos y analizaremos tanto los elementos que actúan como obstaculizadores como los elementos que actúan como facilitadores.

Antes de describir los resultados, nos gustaría recordar y matizar que entendemos los procesos obstaculizadores como aquellos obstáculos que dificultan, obstaculizan y/o limitan sus procesos de aprendizaje no estando, por lo tanto, en condiciones de igualdad respecto a sus compañeros y compañeras $y$, consecuentemente, pudiéndose experimentar situaciones de vulnerabilidad. Respecto a los procesos facilitadores, los entendemos como aquellos procesos que facilitan sus procesos de aprendizaje y fomentan la inclusión dentro de las aulas universitarias.

En las experiencias que presentamos a continuación, vamos a diferenciar el papel del profesorado y la actuación que tienen los proyectos docentes en el aprendizaje de los alumnos con diversidad funcional de la Universidad de Sevilla.

\section{I. El doble papel del profesorado}

Tras el análisis realizado, los II participantes llegan al consenso de que el profesorado aparece en su trayectoria con un doble papel: facilitador y obstaculizador en sus experiencias universitarias.

Debemos de indicar, que, aunque en menor número de representación, se hace más destacado el profesorado que actúa como barrera que el que actúa como facilitador debido a las dificultades y a los problemas de aprendizaje que originan al estudiante. Los estudiantes, reclaman al profesorado una ayuda más humana, personalizada y atenta a sus necesidades, reclamando una mayor sensibilización, atención y apoyo para poder alcanzar con éxito sus objetivos en el aprendizaje. Este requisito ha aparecido en todos los grupos de discusión y entrevistas realizadas, donde los participantes piden un profesorado más competente y formado para atender a la diversidad existente en las aulas.

RSP9: "Yo estoy con RSPIO. El profesorado no se encuentra formado para atender a nuestras necesidades porque tú llegas y no saben qué hacer; tú les dices "mira yo tengo esta discapacidad y necesito estas adaptaciones" y te dicen "bueno ¿y eso cómo lo hago?", y muchas veces, he tenido que ser yo el que le diga cómo tienen que adaptar mis cosas. Es que yo no lo entiendo, no hay formación para atender a las distintas discapacidades. Ya no sólo con mi caso o con el caso de esta mujer, sino con todos los demás." 
En este debate generado, cabría mencionar la aportación que realiza RSP9, quién indica que al inicio del curso, todos los años ha de acudir al despacho de los profesores para informarles sobre su situación personal y necesidades educativas. Según comenta este estudiante, echa en falta la coordinación entre Secretaría y el profesorado, puesto que indica que, en el momento de formalizar la matrícula, el alumno ha de indicar el tipo de discapacidad que tiene y el grado y que, a pesar de ello, esta información no llega nunca al profesorado. Afirman que, si esta información llegara a conocerse por adelantado por parte del profesorado, podría evitarse situaciones como la expuesta anteriormente, pudiendo tener el material de la asignatura accesible y adaptado.

Por otro lado, al preguntar durante los grupos de discusión por el profesorado que menos le gusta, los estudiantes con diversidad funcional de esta investigación, aseguran que son aquellos profesores que se caracterizan por ser teóricos, cuyas clases son magistrales, escaseando la interacción con otros medios más tecnológicos $y$, cuando lo usan, no lo hacen como apoyo en sus explicaciones sino como las explicaciones en sí, es decir, se limitan a leer las transparencias sin aportar ningún ejemplo ni relacionarlo con otro contenido. A este tipo de profesorado, los estudiantes lo consideran como mero transmisor de conocimientos pero no como generador de ellos. Lo señalan como barrera, ejerciendo en ellos una influencia negativa en su participación activa y asistencia a clase, llegando a afirmar que su motivación por el aprendizaje se ve mermada.

Otra de las cuestiones tratadas, es la formación del profesorado frente a la diversidad. Ante esta cuestión, nos hemos encontrado con una respuesta unánime por parte del grupo: el profesorado no se encuentra preparado para responder a las necesidades educativas especiales que los alumnos con diversidad funcional muestran, y lo que ellos y ellas señalan como más asombroso aún, es que les extraña que en carreras de Ciencias Sociales como es el caso de Magisterio, Pedagogía y Psicología tengan esta carencia, considerándose ésta una formación indispensable.

No obstante, los estudiantes también hacen mención a los profesores que han actuado como facilitadores en sus profesos de aprendizaje, $y$, señalan que son la mayoría, aunque no sean tan visibles como los profesores que actúan como obstaculizadores por todas las trabas impuestas y lo que ello les genera.

En el caso de los profesores que han actuado como facilitadores en su proceso de aprendizaje los caracterizan como humanitarios e indican que son un pilar 
importante durante su trayectoria de aprendizaje. Entre las actuaciones que este tipo de profesorado realiza para ayudar a los estudiantes, destacan la adaptación de los materiales impartidos en clase a sus necesidades, además de que éste es facilitado por adelantado durante el desarrollo de las asignaturas; de igual forma matizan y remarcan el uso que este profesorado hace de las Nuevas Tecnologías para impartir su asignatura así como las facilidades que estas ofrecen: tienen el material siempre accesible, pueden seguir el ritmo de la clase, siendo un recurso y apoyo visual-auditivo y facilitando la interacción y comunicación entre profesores y compañeros, etc.

RSP4: "En cuanto al profesorado que actúa como ayuda, no tengo más que destacar al hecho de que me han aportado bastante ayuda y facilidades. En general, este curso, he recibido atención, apoyo y comprensión por parte de los profesores, éstos me han dado los apuntes de clase con tiempo suficiente para poderlos estudiar además de que lo han adaptado a mis necesidades y no sólo durante la asignatura sino también en los exámenes”

Respecto al tipo de profesorado que los estudiantes destacan como ideal, podemos indicar que de manera consensuada, los once participantes coinciden en definir a este profesorado como transmisor y generador de conocimiento, reclamando una combinación de clases teóricas con clases prácticas, proporcionando ejemplos reales y motivándolos por la asignatura incluyendo nuevos elementos de aprendizaje, pero fundamentalmente, lo que piden es un profesorado que les muestre apoyo y comprensión ante sus necesidades, es decir, un profesorado que sea humanitario.

Para finalizar este apartado, vamos a presentar las recomendaciones que estos estudiantes con diversidad funcional realizan al profesorado a través del siguiente planteamiento: "En el futuro, ¿qué le recomendarías a los profesores y profesoras para que sus clases fueran más inclusivas, y por lo tanto, para que todo el alumnado puedan aprender y participar?”. La gran mayoría de los participantes, coinciden en señalar que el profesorado debe de tener una mejor y mayor formación ante los diferentes tipos de discapacidades existentes y que, por lo tanto, esto le permita realizar clases más inclusivas. También señalan, que sería importante desarrollar clases más prácticas y condensar el material y contenido de las asignaturas sin que éstos sean tan excesivos que lleguen a desbordar las capacidades de los estudiantes, además, reclaman que el profesorado tenga sus clases preparadas y trabajadas, pero, sobre todo, lo que piden es que tengan un cambio de mentalidad y mayor sensibilización ante las necesidades individuales que puedan presentar, pudiendo ser éstas tanto personales como académicas. 


\section{2. ¿Cómo son los proyectos docentes: obstaculizadores o facilitadores?}

A grosso modo, se puede identificar como la gran mayoría de los participantes destacan como una barrera inaccesible a su aprendizaje la obligatoriedad de asistencia a las clases, aspecto que le repercute negativamente en sus calificaciones debido a la imposibilidad de asistir por su problemática personal. Indican, que esta situación, no es única ni exclusiva de las personas que tienen discapacidad, sino que este problema lo tienen también muchos compañeros.

Asimismo, los participantes en esta investigación, destacan a un pequeño grupo del profesorado como una barrera al no acceder ni modificar sus proyectos docentes ante las necesidades específicas de apoyo educativo que éstos puedan presentar derivadas de su discapacidad al no realizar las adaptaciones necesarias para poder incluirlos en el aula. Por ejemplo, RSP9 nos explica cómo tuvo problemas con un profesor ya que no podía ver las diapositivas y, por lo tanto, no podía estudiarlas. Ante esto habló con el servicio de la ONCE quién le transmitió este problema, no obstante, nos indica que el profesor hizo caso omiso a esta reclamación, por lo que, decidió ponerse en contacto con el SACU, en concreto con la persona encargada del Servicio de Atención al Discapacitado, y mediante la cual, se solucionó toda la problemática. Esta forma de actuar hace que consideren a ese grupo de profesores demasiados inflexibles y poco atentos a sus necesidades.

RSP4: "Respecto al profesorado, la verdad, me encuentro un poco desilusionado, concretamente con un profesor cuya asignatura era muy visual y se negó a adaptarme el material ni adaptarme las prácticas. Así que, al final, tuve que dejar la asignatura."

Por otro lado, la gran mayoría los estudiantes, comentan la abundancia de material y contenidos que el profesorado ofrece, además de la densidad de trabajo difícil de abordar: exámenes y trabajos tanto grupales como individuales. Señalan la poca innovación existente en los proyectos docentes, repitiéndose los mismos contenidos en diferentes cursos.

Frente a la metodología tradicional y magistral, los once participantes señalan cómo los proyectos docentes que fomentan la participación, en los que el profesorado se involucra en el aula y donde son capaces de extrapolar los contenidos de la materia a la realidad les suponen una ayuda en su aprendizaje:

RSP I0: "Yo destaco a los profesores que ponen ejemplos reales en las clases. Si tú lees en el manual, a lo mejor no lo entiendes ni te llega igual que si te lo expone una persona con ejemplos reales y prácticos." 
No obstante, hay algunas actividades contempladas en los proyectos docentes en las que los participantes no pueden participar de manera activa debido a que sus necesidades y capacidades no son tenidas en cuenta y son obviadas, según comentan, sin mala intención por parte de los profesores. Algunos de ellos, indican que esto no les supone ningún problema personal puesto que lo tienen asimilado, como es el caso de RSP8 y RSPI0; mientras que RSP7 nos indica su sentimiento de desigualdad y exclusión:

RSP7: "Mi problema principal lo encuentro en la organización del aula y de mesas. Por ejemplo, mi aula esta organizada en línea, y en una actividad, necesitábamos ponernos en semicírculo, esto requería de un espacio mayor que estaba en el lugar del profesor. Para llegar a él había que subir un peldaño que yo no podía y, me encontré en una situación donde los compañeros estaban arriba y yo abajo, sola. Me vi, no discriminada pero si forzada al aislamiento y el profesor ni buscó ninguna alternativa para adaptar dicha actividad"

Respecto a los resultados académicos obtenidos, en los diferentes grupos de discusión y entrevistas, podemos encontrar un debate con dos grupos claramente delimitados. Por un lado, encontramos aquellos estudiantes que afirman que su discapacidad no ha influido en absoluto en sus resultados académicos, asegurando que los problemas que han tenido a la hora de estudiar son los mismos que los de cualquier otra persona. Por otro lado, nos encontramos a otro grupo de estudiantes que señalan, que más que afectar su discapacidad en sí en sus resultados académicos, ha sido la falta de organización entre sus necesidades individuales y la cantidad y modalidad de trabajo que las titulaciones les han exigido.

RSP9: "Hombre, a mí lo que me pasa es que mi enfermedad es degenerativa. Antes veía más que ahora y podía estudiar más rápido; ahora, necesito que me faciliten los apuntes por adelantado para poder adaptarlos (aumentar el tamaño de la letra o adaptarlo al braille), $y$, en ocasiones, los profesores no me lo facilitan por lo que no puedo estudiar al mismo ritmo que mis compañeros o, por ejemplo, aquellos profesores que, aún facilitándome el material, es tanta cantidad que no me da tiempo a adaptarlo y poderlo estudiar para el examen. Por lo que, no creo que mi discapacidad afecte a mis resultados pero sí la falta de organización y la cantidad de documentos"

Otra de las cuestiones que se plantearon y se trataron en los grupos, fue el uso y manejo de las Nuevas Tecnologías de la Información y Comunicación (NTIC) en las distintas asignaturas. Se preguntó de manera específica si el profesorado estaba 
empleando y utilizando la plataforma tecnológica (Blackboard) o algún otro recurso tecnológico o repositorio digital. Todos ellos confirmaron que sí, además, afirmaron que este medio les supone un gran apoyo y ayuda en su proceso de aprendizaje, puesto que si algún día no pueden asistir a clase, el temario se encuentra disponible en la plataforma las 24 horas del día y que, a través de este espacio, cuentan con el material completo de la asignatura, lo que les supone una ayuda para poder seguir las explicaciones del profesor, y por lo tanto, las clases.

No obstante, señalaron que en algunas ocasiones, la plataforma ha sido usada como un repositorio de material sin utilizar y sin sacar el máximo rendimiento de las herramientas de comunicación que presenta (chat, foro, correo, etc.).

Para finalizar este apartado, al igual que hicimos en el apartado anterior, vamos a presentar los datos extraídos referente a la prospectiva que el alumnado tiene sobre su aula ideal. Las respuestas a dicho planteamiento fueron muy variopintas y de diversa índole: arquitectónica, física, metodológica, formativa y didáctica; no obstante, nosotros únicamente nos vamos a centrar en las respuestas relativas a los proyectos docentes.

Indican que, su Universidad ideal, sería aquella cuyos proyectos docentes se encontraran adaptados a ritmos "normales" de aprendizaje y que éstos no fueran tan densos, proponiendo incluso que se modificaran asignaturas cuatrimestrales por anuales.

\section{Conclusiones}

En este apartado de conclusiones finales, más que enunciar un listado de los resultados obtenidos, vamos a realizar una reflexión final sobre el proceso de prácticas inclusivas llevadas a cabo en la universidad. Llegados a este punto, nos planteamos la siguiente cuestión: iexisten aulas inclusivas en las facultades de Ciencias de la Educación, Psicología y Filosofía de la Universidad de Sevilla? Si atendemos a las experiencias recopilados en este artículo y las analizamos, podemos encontrar más procesos obstaculizadores que facilitadores, por lo que tratamos de responder a esta cuestión aportando información para una mejor comprensión, mejora del diseño y desarrollo de prácticas inclusivas en la universidad.

Las experiencias reflejadas en esta investigación, nos indican que el profesorado ha actuado tanto como elemento obstaculizador como elemento facilitador. Según este alumnado, los profesores y profesoras pertenecientes al primer grupo, son 
menos cuantiosos que los segundos, sin embargo, se hacen más destacables y tediosos por todas las trabas impuestas que les imposibilita alcanzar de una forma óptima su objetivo de aprendizaje.

Respecto al profesorado que actúa como barrera, los estudiantes nos indican que es aquel que no realiza adaptaciones curriculares según sus necesidades individuales de aprendizaje (material adaptado y/o facilitado por adelantado, adaptaciones metodológicas, más tiempo en los exámenes, exámenes orales, etc.), y, especifican, que debido a ello tienen que trabajar el doble que sus compañeros, procesos que en algunas ocasiones puede conllevar al abandono y desmotivación por parte de los estudiantes ante la asignatura.

Esta conclusión, nos lleva a plantearnos el desconocimiento por parte de un sector del profesorado sobre la actual Ley de Universidad 4/2007, donde en su apartado tercero de la disposición adicional vigésimo cuarta se contempla la obligación de las universidad de promover acciones para favorecer la inclusión y dar respuestas a las necesidades educativas especiales que presenten los estudiantes.

En relación al tipo de profesorado que menos le gusta, los estudiantes con diversidad funcional de las facultades de Ciencias de la Educación, Psicología y Filosofía especifican que es aquel cuyo proyecto docente es demasiado denso y que no se puede abarcar en todo el curso escolar, cuyas clases son magistrales, teóricas y poco preparadas, limitándose a una lectura de diapositivas o manual. Todo esto hace que merme la motivación de los estudiantes por la asignatura.

Por otro lado, encontramos que algunos profesores especifican la obligatoriedad de asistencia sin dar ninguna otra opción alternativa a la misma. Igualmente, los estudiantes indican que muchas clases se rigen por metodologías magistrales, aspecto que no les permite participar en las clases ni desarrollar un proceso íntegro de aprendizaje puesto que no son significativos para ellos, además de aprender muy poco; unido a esto, encontramos además la densidad de material de la asignatura y las actividades poco inclusivas de algunos proyectos, lo que supone procesos obstaculizadores hacia las personas con diversidad funcional. Como aspecto positivo, cabe destacar el uso que se realiza en la mayoría de las asignaturas de las Nuevas Tecnologías en los proyectos docentes como apoyo a la docencia y a los procesos de enseñanza-aprendizaje.

Pero la característica más importante, en la que todo los participantes coinciden, es que no les gusta el profesorado que no se encuentra formado para dar respuesta a la diversidad y que, por consiguiente, no sabe cómo realizar las adaptaciones 
necesarias en su asignatura. En esta línea, Castellana y Sala (2005), Gibbs (2004), Gitlow (1999) o Sharma, Forlin y Loreman (2008) indican que el profesorado ha de estar capacitado y formado para enseñar con eficiencia en las aulas dando respuesta a todas las necesidades individuales de aprendizaje que su alumnado pueda tener, adquiriendo un compromiso frente al mismo.

Por un lado, encontramos un amplio número de experiencias positivas por parte de los estudiantes frente al profesorado que actúa como ayuda. Destacan de ellos que son personas empáticas ante sus necesidades, humanitarias que les apoyan y ayudan en su proceso de enseñanza-aprendizaje, realizando las adaptaciones curriculares que se consideran oportunas.

Trabajos como los de Castellana y Sala (2005) concluyen que se puede favorecer la inclusión en las aulas a través del diseño del currículum de la asignatura. Para ello, estas autoras y otros, como puede ser el caso de Moriña (2004) o Stainback y Stainback (1999), proponen que el currículum ha de estar pensado y elaborado para que todos los estudiantes puedan participar en la asignatura, garantizando así un proceso de enseñanza-aprendizaje en igualdad de oportunidades para todos los estudiantes.

Por otro lado, una de las cuestiones más reiteradas y demandadas por parte de los estudiantes, es la falta de formación y especialización por parte de un sector de profesores, quienes aseguran que saben atender a las necesidades individuales de aprendizaje que puede presentar un estudiante con diversidad funcional. Demandan, ya no sólo la formación especializada, sino una mayor sensibilización ante las necesidades derivadas de su discapacidad pudiendo así, conseguir la finalidad principal de la educación inclusiva, que no es otra que dar una respuesta de manera equitativa y de calidad a todo el alumnado. Desde el planteamiento de una organización inclusiva, en la educación no tiene que haber cabida a ninguna práctica que pueda generar algún proceso de segregación o exclusión.

No obstante, las conclusiones que han aparecido en este trabajo, se ven reforzadas por otras investigaciones tanto nacionales como internacionales, como es el caso de Castellana y Sala (2005), Sánchez Palomino (2009, 20I I), Sánchez y Carrión (20I0), Borland y James (1999), Gitlow (1999), Poussu-Olli (1999), Adams y Brown (2006), Vickerman y Blundell (20I0) o Moswela y Mukhopadhyay (20II). Todo esto nos lleva a concluir sobre la necesidad de asesoramiento y formación que necesita el profesorado para mejorar su respuesta ante la diversidad, considerando únicas a las personas, y que, por lo tanto, la respuesta y adaptaciones que a estas se les haya de dar ante sus necesidades ha de ser también únicas. 


\section{Referencias Bibliográficas}

Adams, M. y Brown, S. (2006). Towards inclusive learning in Higher Education developing curricula for disable students. Abingdon: Routledge.

Ainscow, M. (1999). Understanding the development of inclusive schools. London: Falmer Press.

Ainscow, M. (2004). Desarrollo de las Escuelas Inclusivas. Ideas, propuestas y experiencias para mejorar las instituciones escolares. Madrid: Narcea.

Ainscow, M. (2008). Garantizar que cada alumno es importante: La mejora de la equidad dentro de los sistemas. En J. Gairín y S. Antúnez (Coords.), Organizaciones educativas al servicio de la sociedad (I st ed.), 282-287. Barcelona: Walters Kluwe.

Arana, J., Rodríguez, S. y Meilán, J. (2008). Los servicios de Atención Psicológica en la universidad española: Análisis crítico. Revista de Psicología General y Aplicada, 6I, 265-284.

Arnaiz, P. (2003). Educación inclusiva: una escuela para todos. Málaga: Aljibe.

Barnes, C. (2008). La diferencia producida en una década. Reflexiones sobre la investigación "emancipadora” en discapacidad. En L. Barton (Comp.), Superar las barreras de la discapacidad (pp. 38I-397). Madrid: Morata.

Barton, L. (1998). Discapacidad y Sociedad. Madrid: Morata.

Barton. L. (2008). Superar las barreras de la discapacidad. Madrid: Morata.

Bausela, E. (2004). La función orientadora en el marco de la Universidad. EduPsykhé, 3, 109-120.

Borland, J. y James, S. ( 1999). The Learning Experience of Students with Disabilities in Higher Education. A case study of a UK university. Disability and Society, I4 (I), 85- I0 I.

Castellana Rosell, M. y Sala Bars, I. (2005). La Universidad ante la diversidad del aula. Aula Abierta, 85, 57-84.

Echeíta, G. (2006). Educación para la Inclusión o Educación sin exclusiones. Madrid: Narcea.

Frank, A. W. (20I I). Practicing Dialogical Narrative Analysis. En J. A. Gubrium (Eds.), Varieties of narrative analysis (pp. 33-52). Los Ángeles: Sage Publications.

Gibbs, G. (2004). Mejorar la enseñanza y el aprendizaje universitario mediante estrategias institucionales. Educar, 33, I I-30. 
Gitlow, L. (1999). Individuals with Disabilities and Professional Education Programmes: An analysis of legislation, court rulings and policy. Disability and Society, I 4 (2), 239-248.

Healey, M., Fuller, M., Bradley, A. y Hall, T. (2006). Listening to students: the experiences of disables students of learning at university. En M. Adams y S. Brown. (Eds.), Towards Inclusive Learning in Higher Education: Developing Curricula for Disable Students (pp. 32-43). London: RoutledgeFalmer.

Lavié Martínez, J. M. y Marcelo García, C. (2000). Formación y nuevas tecnologías: Posibilidades y condiciones de la teleformación como espacio de aprendizaje. Bordón, Revista de Pedagogía, 52 (3), 385-406.

MacMillan, J. H. y Schumacher, S. (2005). Investigación educativa: Una introducción conceptual. Madrid: Pearson Educación.

Marcelo, C. (1995). Formación del profesorado para el cambio educativo. Barcelona: PPU.

Marcelo, C. (200I). El Aprendizaje de los Formadores en tiempos de Cambio. La aportación de las redes y el caso de la red andaluza de profesionales de la formación. Profesorado: Revista de Currículum y Formación del Profesorado, 5 (I), 29-44.

Miles, M. B. y Huberman, A. M. (1994). Qualitative data analysis. Beberli Hills, CA: Sage Publications.

Moriña Díez, A. (2004). Teoría y Práctica de la Educación inclusiva. Málaga: Aljibe.

Moriña Díez, A. (2010). Vulnerables al silencio: Historias escolares de jóvenes con discapacidad. Revista de Educación, 353, 667-690.

Moriña, A. y Parrilla, A. (2007). Criterios para la formación permanente del profesorado en el marco de la educación inclusiva. Revista de Educación, 339, 5I 7 539.

Moswela, E. y Mukhopadhyay, S. (20II). Asking for too much? The voices of students with disabilities in Botswana. Disability and Society, 26 (3), 307-3 I 9

Parker, V. (1999). Personal Assistance for Students with Disabilities in HE: The experience of the University of East London. Disability and Society, I 4 (4), 483-504.

Poussu-Olli, H. S. ( 1999). To be a Disabled University Student in Finland. Disability and Society, I4 (I), I03-II3. 
Pujadas, J .J. (2002). El método biográfico: El uso de las historias de vida en ciencias sociales. Madrid: Centro de investigaciones sociológicas.

Sánchez, A. y Carrión, J. J. (2010). Los estudiantes con discapacidad en la Universidad de Almería: Ideas y Actitudes sobre su integración Educativa y Social. European Journal of Education and Psychology, 3, (2), 329-34I.

Sánchez Palomino, A. (2009). Integración educativa y social de los estudiantes con discapacidad en la Universidad de Almería. Almería: Editorial Universidad de Almería.

Sánchez Palomino, A. (20II). La universidad de Almería ante la integración educativa y social de los estudiantes con discapacidad: Ideas y actitudes del personal docente e investigador. Revista De Educación, 354, 32 I-322.

Sandín, M. (2003). Investigación cualitativa en educación. Fundamentos y tradiciones. Barcelona: McGraw Hill.

Sharma, U., Forlin, C. y Loreman, T. (2008). Impact of training on pre-service teachers attitudes and concerns about inclusive education and sentiments about persons with disabilities. Disability and Society, 23 (7), 773-785.

Stainback, S. y Stainback, W. (1999). Aulas inclusivas. Madrid: Narcea.

UNESCO (2008). Educación para todos en 20I5. ¿Alcanzaremos la meta? Informe del seguimiento de la EPT en el mundo. Francia: UNESCO.

Vickerman, P. y Bloundell, M. (2010). Hearing the voices of disabled students in higher education. Disability and Society, 25 (2), 2 I-32.

\section{I. Fuentes Electrónicas}

Ley Orgánica de Universidades 4/2007, de 12 de abril, por la que se modifica la Ley Orgánica 6/200I, de 21 de diciembre, de Universidades. BOE n 89. Recuperado de http://noticias.juridicas.com/base_datos/Admin/lo6-200I.html

Novo Corti, I., Muñoz Cantero, J. M. y Calvo Porral, C. (20II). Análisis de las actitudes de los jóvenes universitarios hacia la discapacidad: un enfoque desde la teoría de la acción razonada. Relieve, I7, (2), art. 5. Disponible en http://www.uv.es/ RELIEVE/vI7n2/RELIEVEvI7n2_5.htm 


\section{Notas}

I. "Barreras y ayudas que los estudiantes con discapacidad identifican en la Universidad”. Proyecto financiado por el MICINN, I+D+I, ref. EDU 20 I0- 16264.

2. Los datos extraídos sobre el número de estudiantes con diversidad funcional matriculados en la Universidad de Sevilla han sido facilitados por la persona responsable del Servicio de Atención al Alumnado Universitario (SACU), más concretamente, en el Dpto. de Atención al Alumnado con Discapacidad.

3. De aquí en adelante, para no reiterarnos, cuando digamos Universidad estaremos haciendo alusión a la Universidad de Sevilla. Si mencionamos cualquier otra Universidad que no sea ésta, haremos mención explícita a la misma.

4. Los cinco campos de conocimientos de la Universidad de Sevilla son: Ciencias de la Salud, Ciencias Experimentales, Ciencias Sociales y Jurídicas, Ingeniería y Tecnología, y, Humanidades.

5. Diseñar una guía de formación online para el profesorado universitario con el fin de dar respuesta a las necesidades educativas del alumnado con diversidad funcional.

6. La población del estudio, es decir, el alumnado con diversidad funcional de la Universidad de Sevilla se encuentra compuesta por 445 estudiantes matriculados durante el curso 09/10.

7. Como se ha indicado al inicio del artículo, los datos presentados en el mismo únicamente se refieren a las Facultades de Ciencias de la Educación, Psicología y Filosofía, de ahí que la muestra sea de I I participantes.

8. Sobre dicha población se identificaron 86 estudiantes en las Facultades de Ciencias de la Educación, Psicología y Filosofía (datos facilitados por el Servicio de Atención a las Personas con Discapacidad), de los cuales tan sólo I I fueron los que respondieron a la invitación del proyecto

9. EI SACU fue el encargado de dar a conocer vía email entre la población universitaria con diversidad funcional el proyecto que el equipo de investigación estaba llevando a cabo y fueron las personas interesadas en participar quiénes se pusieron en contacto con la responsable de la Unidad de Atención a las Personas con Discapacidad y los investigadores. 